\title{
A NEW INDEX OF THE INTRAPULMONARY MIXTURE OF INSPIRED AIR
}

\author{
BY
}

MARGARET R. BECKLAKE

From the Department of Medicine, Postgraduate Medical School of London, and the Cardio-Pulmonary Unit, C.S.I.R. Department of Medicine, University of the Witwatersrand

(RECEIVED FOR PUBLICATION OCTOBER 31, 1951)

In numerous studies it has been shown that the emphysematous lung is unevenly ventilated, the inspired air being more effectively mixed into some parts than into others, and it has been usual to express the results of such studies graphically. Some workers have emphasized the importance of the shape of the graph (Briscoe, Becklake, and Rose, 1951), whereas others have expressed the efficiency of intrapulmonary mixing as indices related to one or other of the variables upon which the shape of the graph depends (Bateman, 1950; Bates and Christie, 1950 ; Birath, 1944 ; Darling, Cournand, and Richards, 1944).

One such index is that of Cournand, Baldwin, Darling, and Richards (1941). These workers had noticed that the alveolar nitrogen content after seven minutes of pure oxygen breathing was higher in emphysematous than in normal subjects. This suggested that in emphysematous subjects the tidal air was less effective in washing out the alveolar spaces. They adapted their open-circuit method of measuring lung volume to measure the efficiency of the intrapulmonary distribution of inspired air (Darling, Cournand, and Richards, 1944), expressing their results in terms of the difference between the observed and the predicted alveolar nitrogen content after various numbers of breaths. Their prediction formula was based on the assumption that in normal subjects a volume of air equal to the tidal air minus the anatomical dead space was evenly distributed to all lung spaces. Because this method was too laborious for routine use, they subsequently (Baldwin, Cournand, and Richards, 1948) reverted to their original method of measuring only the alveolar nitrogen content after seven minutes of pure oxygen breathing. The theoretical objections to this figure as a mixing index are, as they pointed out, that it does not allow for variations in minute ventilation or functional residual air.

Another index was suggested by Birath (1944), who observed the rate at which equilibration took place between the lungs and a closed spirometer system containing hydrogen as the indicator gas. From the mixing curve he estimated "dead space," using the ratio between this physiological "dead space" and the mean tidal air as an index of intrapulmonary mixing efficiency. Measurements of "dead space" have little meaning when the lung is unevenly ventilated, as the dead space appears to increase during the course of a single mixing study after the well-ventilated parts of the lung have equilibrated (Becklake, 1951 ; Briscoe, Becklake, and Rose, 1951). Hence it would be misleading to represent the "dead space" of such 
a lung as the mean of a range of values. The same objection applies to a similar index used by Bateman (1950), who employed an open-circuit technique. In fact this worker discarded all cases in which the mixing curves of normal subjects showed different dead spaces at different stages of the mixing process, thus in effect excluding all subjects with uneven intrapulmonary ventilation (Robertson, Siri, and Jones, 1950).

A more logical index of intrapulmonary mixing has recently been described by Bates and Christie (1950), who suggested that, using a closed-circuit apparatus, mixing efficiency could usefully be assessed as the ratio between the expected and the observed number of breaths needed to reach the point of $90 \%$ mixing. The expected number of breaths was calculated on the assumption that the whole of each breath was evenly distributed throughout the lungs, no allowance being made for the dead space of their apparatus (which was negligible) or the dead space of the patient. When applied to normal and emphysematous subjects this index gave a good separation between the two groups.

\section{The "Lung Clearance Index"}

It seemed that a simpler index might be provided by an estimate of the litres of ventilation required to wash each litre of the functional residual air (F.R.A.) free of nitrogen while the subject breathes pure oxygen. This has been named the "lung clearance index" and is calculated as follows :

$$
\frac{\text { Litres ventilation required to wash } 90 \% \text { F.R.A. free of } \mathrm{N}_{2}}{90 \% \text { F.R.A. }}
$$

The $90 \%$ mixing point was selected for study because the shape of the mixing curve was such that this point could be more accurately determined than the moment of complete mixing. (See also Bates and Christie, 1950.)

This paper reports a study of this index, and of those of Cournand, of Birath, and of Bates and Christie in normal and emphysematous subjects. The open-circuit nitrogen clearance technique previously described (Becklake, 1951) was used. The index of Cournand and the lung clearance index are the only two directly applicable to an open-circuit technique. Birath (1944) and Bates and Christie (1950) used closed-circuit methods, but their indices have been adapted to describe the results obtained with an open-circuit technique.

In the results index 1 refers to the index of Baldwin, Cournand, and Richards (1948), index 2 to that of Birath (1944) modified for the open circuit, index 3 to that of Bates and Christie (1950) similarly modified, and index 4 to the lung clearance index.

\section{MATERIAL}

Fifty-nine mixing studies were performed on 51 subjects, who fell into two groups.

Normal Subjects.-Twenty-eight studies were performed on 23 normal subjects in whom history and clinical examination indicated freedom from chest disease. Their ages ranged from 15 to 80 years, 10 being over 35 years of age. The group included ambulant, healthy, normal subjects and convalescent hospital patients with non-respiratory complaints-e.g., peptic ulcer, gastro-enteritis.

Emphysematous Subjects.-Thirty-one studies were performed on 28 emphysematous subjects whose ages ranged from 14 to 70 years. The diagnosis of emphysema was made 
on a history of chronic cough with expectoration and dyspnoea. In five subjects bronchospasm due to asthma dominated the clinical picture. Most of the patients showed cyanosis and had the characteristic configuration of the chest, -together with radiographic appearances of emphysema. In some cases further confirmation of the diagnosis was obtained from electrocardiograms, direct measurement of right ventricular pressures, and the arterial oxygen saturation.

In addition to the clinical assessment and radiological examination, both of which may be inconclusive and misleading (Christie, 1944), the following tests of lung function were performed in all cases to ensure as accurate an assessment of each as possible : spirometry, with scrutiny of the tracings for the characteristics commonly seen in emphysema (Baldwin, Cournand, and Richards, 1948 ; Cournand, Richards, and Darling, 1939) ; measurement of total lung volume and its subdivisions and of the maximum breathing capacity, and comparison with the predicted values (Baldwin, Cournand, and Richards, 1948).

\section{RESULTS}

The results of the pulmonary function tests other than the mixing indices confirmed the clinical assessment of the cases. They are summarized in Table I.

TABLE I

Lung Volumes and Subdivisions and Maximum Breathing Capacities in 23 Normal AND IN 28 EMPHYSEMATOUS SUBJECTS

\begin{tabular}{|c|c|c|c|c|c|c|c|c|c|}
\hline & \multicolumn{6}{|c|}{ Normal Subjects } & \multirow{2}{*}{\multicolumn{3}{|c|}{$\begin{array}{l}\text { Emphysematous } \\
\text { Subjects }\end{array}$}} \\
\hline & \multicolumn{3}{|c|}{ Under 35 years } & \multicolumn{3}{|c|}{ Over 35 years } & & & \\
\hline & Mean & Range & S.D. & Mean & Range & S.D. & Mean & Range & S.D \\
\hline Vital capacity* $\quad$.. & 95 & $\begin{array}{r}69 \\
130\end{array}$ & $14 \cdot 7$ & 87 & $\begin{array}{r}55 \\
133 \\
\end{array}$ & $28 \cdot 5$ & 68 & $\begin{array}{r}38 \\
115 \\
\end{array}$ & $17 \cdot 2$ \\
\hline Total lung volume* & 112 & $\begin{array}{r}78 \\
144\end{array}$ & $18 \cdot 9$ & 108 & $\begin{array}{r}72 \\
150\end{array}$ & $24 \cdot 7$ & 108 & $\begin{array}{r}51 \\
135\end{array}$ & $18 \cdot 3$ \\
\hline 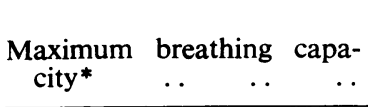 & 93 & $\begin{array}{r}74 \\
118 \\
\end{array}$ & $13 \cdot 3$ & 79 & $\begin{array}{r}48 \\
147 \\
\end{array}$ & $36 \cdot 1$ & 53 & $\begin{array}{l}30 \\
91 \\
\end{array}$ & $18 \cdot 7$ \\
\hline Functional residual air $\dagger \quad \ldots$ & 55 & $\begin{array}{l}41 \\
62\end{array}$ & $5 \cdot 7$ & 61 & $\begin{array}{l}54 \\
72\end{array}$ & $7 \cdot 2$ & 73 & $\begin{array}{l}51 \\
90\end{array}$ & $8 \cdot 0$ \\
\hline Residual air $\dagger$ & 29 & $\begin{array}{r}19 \\
34\end{array}$ & $4 \cdot 9$ & 41 & $\begin{array}{l}18 \\
48\end{array}$ & $10 \cdot 3$ & 57 & $\begin{array}{l}34 \\
76\end{array}$ & $9 \cdot 6$ \\
\hline
\end{tabular}

* Expressed as percentage of predicted value.

+ Expressed as percentage of total lung volume

In the group of normal subjects under 35 years of age, the figures for vital capacity, total lung volume, maximum breathing capacity, functional residual air, and residual air fell within the normal range, and in no case did the spirogram show any features characteristic of emphysema. For normal subjects over 35 years of age the mean figures for vital capacity and maximum breathing capacity, though 
slightly lower than those for the younger subjects, were not remarkable. However, the mean percentages of functional residual air and residual air of the total lung volume were rather high, and two subjects showed the characteristic spirographic features of emphysema. The subjects whose tests showed most deviation from normal were hospital patients convalescent from-non-respiratory illnesses. In none was there any history of dyspnoea, or of chronic cough with expectoration, nor were the clinical findings suggestive of emphysema. These cases form the best basis for comparison with the emphysematous group, who were for the most part over 35 years of age and were hospital patients.

In the majority of emphysematous cases, all tests supported the diagnosis, and in the few cases where one test was within normal limits the other tests were all abnormal. In this group, the mean figures for vital capacity, total lung volume, and maximum breathing capacity expressed as percentages of their predicted values were $68 \%$ (range 38-115), 108\% (range 51-135), and 53\% (range 30-91) respectively, and for functional residual air and residual air as percentages of total lung volume $73 \%$ (range 51-90) and 57\% (range 34-76) respectively. In five cases only were the spirograms quite normal.

Results of mixing studies are set out in Fig. 1 with all four indices for each subject in the same vertical column. It will be seen that indices 3 and 4 give much better separation between emphysematous and normal subjects than do indices 1 and 2.

Index 1 (Baldwin, Cournand, and Richards, 1948).-According to this index one normal subject (No. 10) falls outside the normal range (i.e. over $2.5 \%$ nitrogen in alveolar air after seven minutes of oxygen breathing) whereas by other indices he falls in the borderline between the normal and emphysematous group. His tidal air during the test was low $(0.4381$.$) , and it is probable that index 1$ is abnormal simply because of his shallow breathing. This illustrates one of the greatest shortcomings of the index, namely that it fails to take into account the size of the tidal air. The same defect of this index is demonstrated by the fact that the eight cases of emphysema (Nos. 1, 2, 9, 11, 16, 24, 25, 26) in which this index is normal, but in which all other indices suggest impaired mixing, maintained rather high tidal ventilation throughout the test $(0.578$ to 1.1501 .). Thus index 1 may give false results, both normal and abnormal.

Index 2 (Birath, 1944). - The theoretical objections to this index have been mentioned, and in the present study it gave a poor separation between the normal and the emphysematous groups. The mean value for the normal group was $41 \%$ (S.D. 7.2) and the observed range 27 to $54 \%$. Thus, only in cases with an index over $55.4 \%$ (i.e. mean +2 S.D.) was there a high chance that the index was abnormal. Since at least 16 of the emphysematous cases fell within the upper limit of normal so defined, this index was unreliable in distinguishing the normal from the emphysematous subjects.

Birath's results on normal subjects showed a mean of $32 \%$ (range $18-44 \%$ ) and his emphysematous group a mean of $55 \%$ (range $38-76 \%$ ), and he advised an arbitrary figure of $50 \%$ as the upper limit of normal. One possible reason for his lower figure for normal is that his normal series did not include any subjects over 40 years of age, whereas the present series included a number of older subjects in 


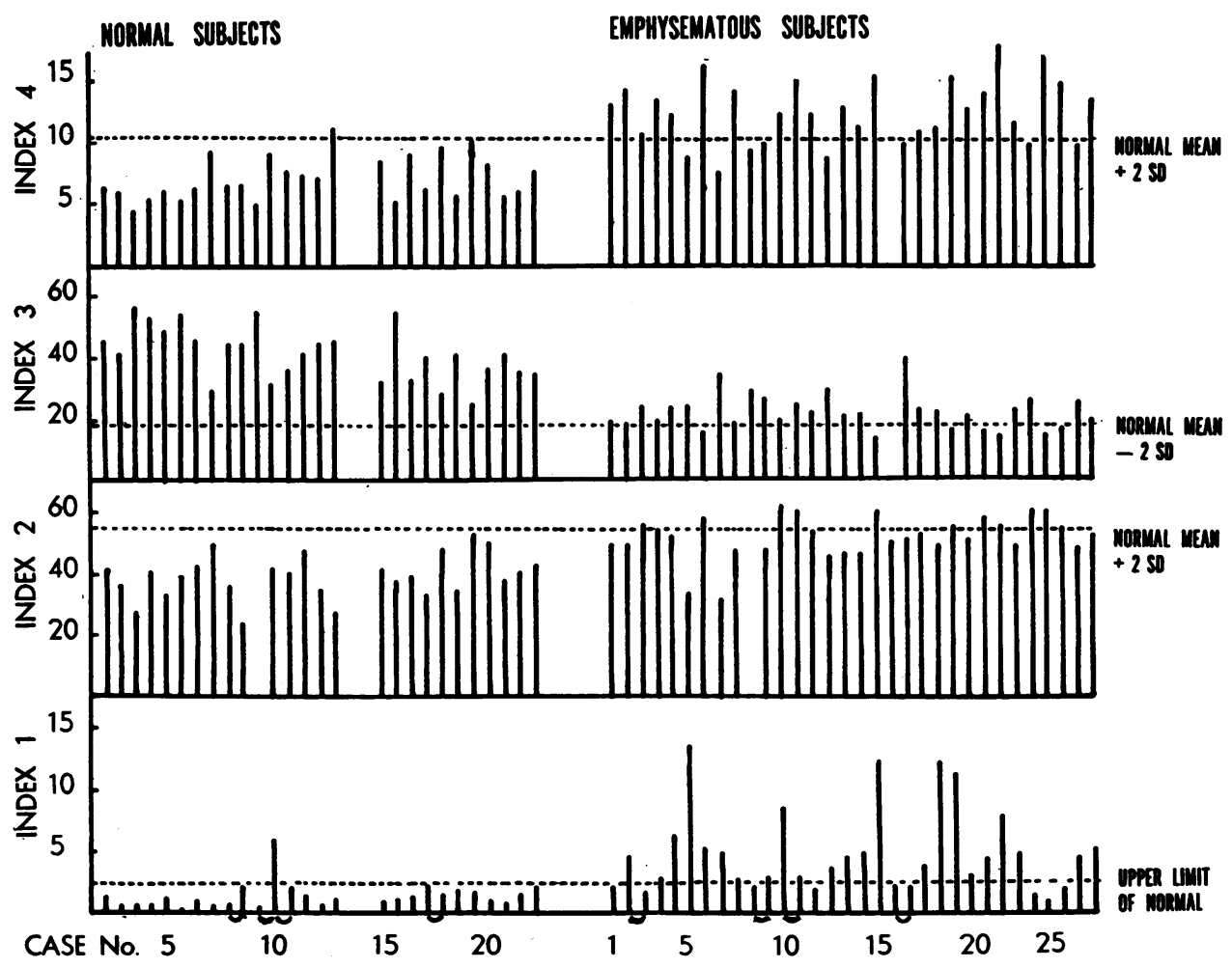

FIG. 1.-Lung mixing indices on normal and emphysematous subjects. Four mixing indices for each subject are plotted in the same vertical column. Index 1 is the index of Baldwin, Cournand, and Richards; index 2 is calculated on the principle suggested by Birath; index 3 is after the style of the index of Bates and Christie; index 4 is the lung clearance index. The dotted lines indicate the mean normal figure for each index +2 S.D.

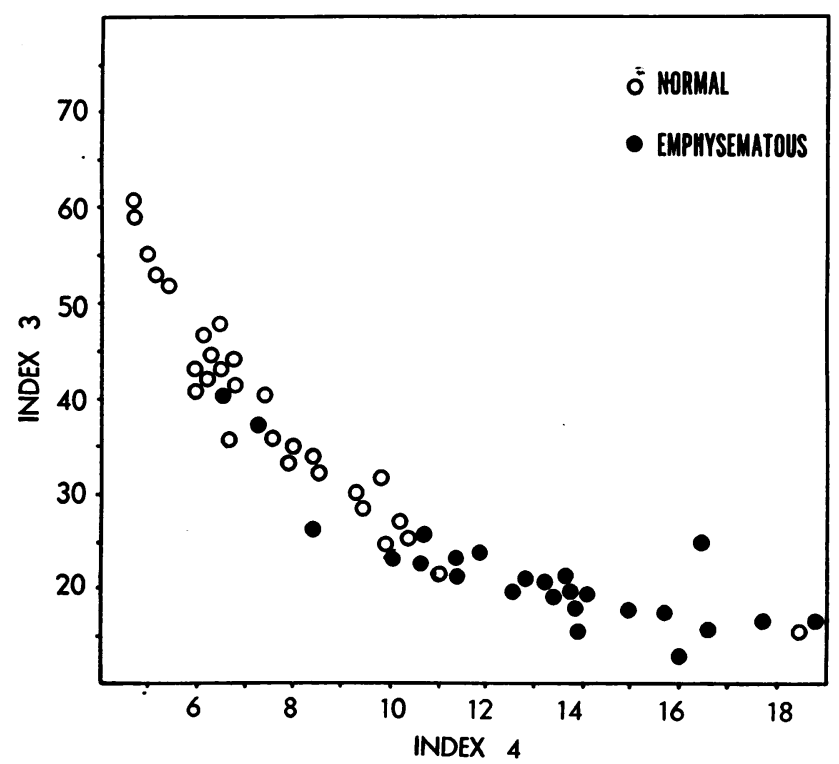

Fig. 2.-Comparison of index 3 (after Bates and Christie) with index 4 (lung clearance index). 
whom there may be some impairment of intrapulmonary mixing (Bates and Christie, 1950 ; Darling, Cournand, and Richards, 1944 ; Robertson, Siri, and Jones, 1950).

Indices 3 (Bates and Christie, 1950) and 4 (Lung Clearance Index).-From Fig. 1 it can be seen that both these indices give a fair separation between the normal and emphysematous groups. A direct comparison between them is shown in Fig. 2, which illustrates graphically an advantage of index 4, namely that the normal subjects are ranged closely together whereas the emphysematous subjects show a wide range. In contrast, index 3 shows a wider range for the normal subjects, thereby reducing its value as a diagnostic test. The mean of the normal group (index 3) was $40.6 \%$ (S.D. 10.93) ; thus, only in cases with indices below $18.74 \%$ (i.e. mean -2 S.D.) was there a strong chance that intrapulmonary mixing was impaired. Twenty-three of the emphysematous cases did not in fact have indices below this critical level. On the other hand, the close grouping of the normals as calculated by index 4 gave a standard deviation of \pm 1.68 about the mean of 7.02. For this index, figures greater than 10.38 are likely to indicate impaired intrapulmonary mixing with a significant difference from the normal. Only eight cases of emphysema fall below this level. As judged by this series of cases index 4 is likely to be of greater value in the diagnosis of emphysema than index 3.

In the group of emphysematous subjects, eight had lung clearance indices of less than 10.38. Four of these (Nos. 10, 17, 24, 27) were on the borderline, having indices between 10 and 10.38, and in these four the tidal air was large. If allowance were made for the anatomical dead space the results for these subjects might have fallen within the emphysematous range.

The other four subjects (Nos. 5, 7, 9, 12) had indices which fell well within the normal range. It is possible that these çases belong to a group originally described by Darling and others (1944) and subsequently reported by Birath (1944) in which there is an increase in the relative and absolute volume of the functional residual air and the residual air, but in which the tidal air is nevertheless evenly distributed to the lung spaces.

\section{SUMMARY}

The value of various indices of intrapulmonary mixing is discussed, and a new index called the lung clearance index is suggested.

Mixing studies on 23 normal and 27 emphysematous subjects are reported. In each case the results are variously expressed by four indices. Of these indices, the lung clearance index is the simplest and most satisfactory in separating normal from emphysematous subjects.

\section{REFERENCES}

Baldwin, E. deF., Cournand, A., and Richards, D. W. (1948). Medicine, Baltimore, $27,243$.

Bateman, J. B. (1950). J. appl. Physiol., 3, 143.

Bates, D. V., and Christie, R. V. (1950). Clin. Sci., 9, 17.

Becklake, M. R. (1951). Thorax, 6, 433.

Birath, G. (1944). Acta med. scand., suppl. 154.

Briscoe, W. A., Becklake, M. R., and Rose, T. F. (1951). Clin. Sci., 10, 37.

Christie, R. V.(1944). Brit. med. J., 1, 105.

Cournand, A., Baldwin, E. deF., Darling, R. C., and Richards, D. W. (1941). J. clin. Invest., $20,681$. Richards, D. W., and Darling, R. C. (1939). Amer. Rev. Tuberc., 40, 487.

Darling, R. C., Cournand, A., and Richards, D. W. (1944). J. clin. Invest., $23,55$.

Robertson, J. S., Siri, W. E., and Jones, H. B. (1950). Ibid., 29, 577. 